\title{
The learning curve of laparoscopic inguinal hernia repair: a comparison of three inexperienced surgeons
}

\author{
Beslen Goksoy ${ }^{1}$, Ibrahim Fethi Azamat ${ }^{1}$, Gokhan Yilmaz ${ }^{2}$, Ozlem Zeliha Sert ${ }^{3}$, Ender Onur ${ }^{1}$ \\ ${ }^{1}$ Department of General Surgery, Sancaktepe Şehit Prof Dr Ilhan Varank Training and Research Hospital, University of Health Sciences, \\ Istanbul, Turkey \\ ${ }^{2}$ Department of General Surgery, Istanbul Medipol University, Istanbul, Turkey \\ ${ }^{3}$ Department of General Surgery, University of Health Sciences, Haydarpasa Education and Research Hospital, Istanbul, Turkey
}

Videosurgery Miniinv 2021; 16 (2): 336-346

DOI: https://doi.org/10.5114/wiitm.2020.100831

\begin{abstract}
Introduction: Studies with inexperienced surgeons in terms of the learning curve for laparoscopic totally extraperitoneal (TEP) inguinal hernia repair are limited.

Aim: To compare three inexperienced surgeons in terms of the learning curve without supervision.

Material and methods: Patients' data, which were from consecutive laparoscopic TEP hernioplasties between December 2017 and February 2020, were analysed retrospectively. The primary outcome was to compare the learning curve of three surgeons (Surgeon A, B, and C) in terms of complications, conversion, and duration of surgery. Secondary outcomes were recurrence rates.

Results: A total of 299 patients were included in the study. Conversion and intraoperative complication rates decreased after the first 60 cases (from 10\% to 2.5\%, $p=0.013$ and from 9\% to 2.5\%, $p=0.027$, respectively). The mean operative time reached a plateau of less than 40 min after 51-81 cases (Surgeon A 51, B 71, and C 81 cases). Ageing was a risk factor for intraoperative complications and recurrence ( $p<0.001, p=0.008$, respectively), and higher body mass index (BMI) was a risk factor for conversion $(p=0.004)$. Age $\geq 60$ years compared to age $<60$ years increased intraoperative complications five-fold and recurrence six-fold ( $p=0.001)$. On the other hand, BMI $\geq 30 \mathrm{~kg} / \mathrm{m}^{2}$ increased the possibility of conversion to open surgery nine-fold $(p<0.001)$. In addition, a positive correlation was found between the operative time and the BMI and VAS score ( $p=0.004, p=0.015$, respectively).

Conclusions: In order to reach the plateau in the operative time during the TEP learning curve period, more than 50 cases should be experienced, whereas more than 60 cases are needed for conversion, intraoperative complications, and recurrence.
\end{abstract}

Key words: laparoscopy, totally extraperitoneal, inguinal hernia, learning curve, transabdominal preperitoneal.

\section{Introduction}

Ralph Ger described the first potential laparoscopic inguinal hernia repair in 1982, but the popularity of the procedure began in the 1990s [1, 2]. Afterward, in 1992, Ferzli et al. defined laparoscopic extraperitoneal inguinal hernia repair and today's total extraper- itoneal hernioplasty for the first time (TEP) [3]. Like all laparoscopic surgeries, laparoscopic TEP provides less postoperative pain and faster recovery. It also has lower recurrence rates than open surgery (Lichtenstein's) [4]. The learning curve of TEP is longer than the transabdominal preperitoneal (TAPP) approach, due to the dissection solely in preperitoneal space

\section{Address for correspondence}

Beslen Goksoy, Department of General Surgery, Sancaktepe Şehit Prof Dr Ilhan Varank Training and Research Hospital,

University of Health Sciences, Istanbul, Turkey, e-mail: beslengoksoy@gmail.com 
and limited area of work which the surgeon is not familiar with [5]. TEP repair uses the Stoppa approach, which advocates the preperitoneal approach, and the Lichtenstein technique, which is a tension-free way of repair [6, 7]. Compared to open surgery, laparoscopic hernia repair has become more popular today due to the advantages mentioned above. However, this method also has some disadvantages such as longer surgery time and a higher risk of complications until completing the learning curve [8-10].

In this process, the performance of a surgeon can be evaluated with the learning curve. The concept of the learning curve was first described by the German physician Hermann Ebbinghaus [11]. Although it is not an accepted standard for the definition or measurement of the learning curve, the surgical learning curve is defined by the number of cases required to perform a specific surgical procedure within a reasonable operative time, with acceptable complication rates and clinical outcomes [12]. In studies conducted in the literature, it has been reported that between 30 and 80 cases are required to gain experience in the laparoscopic TEP learning curve [13-15]. The surgeon's experience, operating room conditions, nurse's experience, and the difference in materials (mesh, etc.) are the most critical reasons affecting the learning curve and may explain this wide range of learning curves in previous studies. To the best of our knowledge, this is the first study in which a group of surgeons are simultaneously undergoing a learning curve in the same centre.

The primary aim of this study was to compare the learning curve of three inexperienced surgeons who perform TEP repair without the supervision of a surgeon experienced in laparoscopic hernia surgery in terms of complications, conversion, and operative time. The secondary aim was to identify the recurrence rates. In addition, operative time, complications, risk factors for conversion and recurrence were examined.

\section{Aim}

This study objective was to compare three inexperienced surgeons under the same conditions in terms of the learning curve without supervision.

\section{Material and methods}

The demographic and clinical data of patients undergoing TEP hernioplasty between 10 December
2017 and 28 February 2020 were retrospectively analysed from the hospital database. Patients with unilateral inguinal hernia (inguinal and femoral), over 18 years old, who were operated electively were included in the study. Patients with bilateral and giant scrotal hernias, recurrent hernias, history of abdominal surgery (median laparotomy and Pfannenstiel incision), patients with a large burn scar in the abdominal region, and patients with strangulated hernia requiring urgent surgery were excluded from the study. The European Hernia Society (EHS) classification system was used in the study [16]. Combined inguinal hernia was defined as a combination of direct and indirect inguinal hernia. The patients' demographic characteristics, hernia type, the operative time, the length of stay in hospital, intraoperative and postoperative complications, conversion (open or TAPP), and recurrence rates were evaluated. Patients with body mass index $\left(\mathrm{BMI} \geq 30 \mathrm{~kg} / \mathrm{m}^{2}\right)$ were defined as obese, and patients $>60$ years old were defined as elderly. The patients were diagnosed with ultrasonography and physical examination before the operation. All patients were operated under general anaesthesia. Antibiotic prophylaxis was not applied. The patients were discharged on postoperative day 1 or 2 . The patients were invited to the outpatient clinic control on the postoperative $10^{\text {th }}$ day, sixth week, sixth month, and 1 year later, and the data were recorded.

The operative time was defined from the first skin incision to the last suture. Intraoperative complications were defined as vascular injury (epigastric or testicular artery or ductus deference) or organ injury or peritoneal rupture requiring sutures. Postoperative complications of haematoma or seroma were defined as blood or fluid accumulation in any subcutaneous tissue area to the scrotal region. Urinary retention was evaluated as the need to use a foley catheter to urinate postoperatively. Chronic groin pain was defined as disturbing moderate pain that lasts at least 3 months after surgery and affects daily activities that decrease over time. Early recurrence was defined as recurrence occurring within the first 6 weeks. The surgeon who performed the surgery decided, in favour of the patient, whether TAPP or open surgery should be chosen when conversion was required. Recurrent patients were identified with clinical examination and radiological imaging (ultrasonography or computed tomography). All patients were operated by three surgeons, Surgeon A, 
Surgeon B, and Surgeon C, who were experienced in laparoscopy (laparoscopic cholecystectomy $n>500$ ) and had performed more than 300 Lichtenstein inguinal hernia repairs but without laparoscopic TEP experience and without a mentor. Before the study the surgeons attended laparoscopic hernia surgery (TEP and TAPP) courses and were certified. All surgeries were performed using the same technique. Each group was divided into 10 subgroups according to the cases, from the first case to the last case. All procedures applied to subjects following the Helsinki Declaration of 1964 and the subsequently published healing principles, and informed consent was obtained from all patients included in the study. The local Ethics Committee approved this study.

\section{Surgical technique}

TEP hernioplasty was performed using threeport techniques. To create the preperitoneal space, a $15 \mathrm{~mm}$ horizontal skin incision was made just below the umbilicus, and the anterior rectus sheath was reached. The anterior rectus sheath was passed through a small incision, and the back of the rectus muscle was reached. After proceeding to the symphysis pubis in the preperitoneal area with the balloon dissector (10-12 mm Covidien ${ }^{\mathrm{TM}}$ Spacemaker ${ }^{\mathrm{TM}}$ Plus Dissector system, USA), the trocar balloon was inflated 20-25 times under the vision of the camera. The balloon trocar was removed, and after 12$14 \mathrm{~mm} \mathrm{Hg}$ pressure $\mathrm{CO}_{2}$ insufflation for pneumoperitoneum, the Hasselbach triangle was examined by exploring the groin with a $10 \mathrm{~mm} 45^{\circ}$ telescope. Afterward, a total of two $5 \mathrm{~mm}$ working ports were inserted, one of which was $2-3 \mathrm{~cm}$ above the symphysis pubis and one between the two ports. Dissection was completed until the spina iliaca anterior superior was in the lateral and the symphysis pubis in the medial, with the epigastric vessels seen in the superior.

The myopectineal orifice was evaluated for potential hernia presence; if a hernia was detected, the hernial sac was reduced using laparoscopic working instruments and bimanual dissection. In the case of peritoneal defects, it was closed with laparoscopic sutures (pre-prepared Endoloop ligation or endoscopic sutures). The gonadal vessel and the vas deference were parietalised in male patients. After the peritoneal hernia defect was dissected up to $3-5 \mathrm{~cm}$ below, a polypropylene patch $(15 \times 12 \mathrm{~cm}$ Covidien
Parietene $^{\mathrm{TM}}$ Macroporous Mesh, USA) was inserted through the camera port so as not to fold over the defect area. It was fixed to the Cooper, the midline rectus muscle, and the iliopubic tract's superior by an endoscopic tacker ( $5 \mathrm{~mm}$ Covidien $^{\mathrm{TM}}$ AbsorbaTack $^{\mathrm{TM}}$, USA). Trocars were removed after desufflation was completed under visual control. In the case of pneumoperitoneum, intra-abdominal gas was evacuated with a Veress needle. The fascia was sutured with $2 / 0$ vicryl suture at the umbilicus incision, and for other trocar sites, only skin incisions were sutured with appropriate sutures.

\section{Statistical analysis}

NCSS (Number Cruncher Statistical System) 2007 (Kaysville, Utah, USA) software was used for statistical analysis. Descriptive statistical methods (mean, standard deviation, median, first quarter, third quarter, frequency, percentage, minimum, maximum) were used when evaluating the study data. The Shapiro-Wilk test and graphical examinations were used to test the suitability of quantitative data for normal distribution. Independent groups $t$-test was used for comparisons of two groups with normal distribution of quantitative variables, and the Mann-Whitney $U$ test was used for comparisons between two groups with non-normally distributed quantitative variables. ANOVA was used for comparison, followed by Bonferroni-correction to compare the normally distributed quantitative variables between more than two groups. Kruskal-Wallis test and Dunn-Bonferroni test were used to compare more than two groups of quantitative variables that did not show normal distribution. Pearson $\chi^{2}$ test, Fisher's exact test, and Fisher-Freeman-Halton exact test were used to compare qualitative data. Pearson correlation analysis was used to evaluate the relationships between quantitative variables. Statistical significance was accepted as $p<0.05$ (Table I).

\section{Results}

Laparoscopic TEP hernia repair was performed in a total of 299 patients ( 80 women, 219 men). Surgeon A operated $34.4 \%(n=103)$ of the patients, Surgeon B 32.8\% $(n=98)$, and Surgeon C 32.8\% $(n=98)$. Most of the cases were lateral hernias. Median follow-up was 19 months (7-28). Thirteen (4.3\%) patients were converted to open surgery, and $8(2.7 \%)$ patients were converted to TAPP. Also, 
$5(1.7 \%)$ of the patients had early and $13(4.3 \%)$ had late recurrence. There was no statistically significant difference in terms of age, BMI, and the length of stay in hospital among groups.

As a result of the evaluations, it was determined that the operative time of the Surgeon A group was lower than the Surgeon B and C groups ( $p=0.025$, $p<0.001$, respectively). Similarly, the VAS scores of the Surgeon A group were lower than the Surgeon B and $C$ groups $(p<0.001)$.

The follow-up time was longer in the Surgeon A group compared with the other two groups ( $p<0.001)$. On the other hand, it was determined that the follow-up time was longer in the Surgeon B group than in the Surgeon C group ( $p<0.001)$. When comparing the parameters among the three groups, no significant differences were found in terms of gender, hernia type, and ASA score. The groups were also similar in terms of conversion to open surgery, conversion to TAPP, intraoperative complications, postop haematoma, seroma, chronic pain, and early and late recurrence rate. A comparison of the groups in terms of clinical characteristics and results are detailed in Table II.

The percentage of conversion (to open surgery or TAPP) was $10 \%$ in the first 60 operations, while this value decreased to $2.5 \%$ after the $60^{\text {th }}$ operation ( $p=0.013)$. Similarly, intraoperative complications decreased from $9 \%$ in the first 60 operations to $2.5 \%$ after the $60^{\text {th }}$ operation $(p=0.027)$.

The rate of conversion to open surgery was statistically significant in patients with higher BMI $(p=0.004)$. When the groups were evaluated among themselves, it was observed that patients who converted to open surgery in the Surgeon $C$ group had higher BMI ( $p=0.009)$ (Table III). In patients with $\mathrm{BMI} \geq 30 \mathrm{~kg} / \mathrm{m}^{2}$, the probability of conversion to open surgery was nine-fold higher than in those with $\mathrm{BMI}<30 \mathrm{~kg} / \mathrm{m}^{2}(\mathrm{OR}=8.944,95 \% \mathrm{Cl}: 2.834-28.225$, $p<0.001)$.

The age of patients with intraoperative complications was similarly higher in all three groups ( $p<$ 0.001) (Table IV). In patients aged $\geq 60$ years, the probability of intraoperative complications was fivefold higher than those of age $<60$ years $(O R=5.262$, 95\% Cl: 1.977-14.003, $p=0.001)$. No major complications were observed in any of our patients.

Total recurrence was seen in $18(6 \%)$ patients, $14(4.6 \%)$ of them in the first 60 cases, and 4 (1.4\%) of them after 60 cases. There was no difference be-
Table I. Magnitude of r. Evans, J. D. (1996). Straightforward statistics for the behavioural sciences. Pacific Grove, CA: Brooks/Cole Publishing

\begin{tabular}{|lc|}
\hline$r$ & Interpretation \\
\hline $0.00-0.19$ & Very weak \\
\hline $0.20-0.39$ & Weak \\
\hline $0.40-0.59$ & Moderate \\
\hline $0.60-0.79$ & Strong \\
\hline $0.80-1.00$ & Very strong \\
\hline
\end{tabular}

tween the three groups. When factors affecting early recurrence were examined, it was observed that age, $\mathrm{BMI}$, and hernia type did not have any effect.

When factors affecting late recurrence were examined, it was found that patients with late recurrence were older $(p=0.008)$. Patients with late recurrence in the Surgeon A group were statistically found to be older than patients in the other groups $(p=0.028)$ (Table $\mathrm{V})$. The probability of late recurrence was six-fold higher in patients over 60 years of age than in patients under 60 years of age $(O R=$ 5.952, 95\% Cl: 1.894-18.706, $p=0.001$ ).

There was a statistically significant relationship between BMI values and operative time $(r=0.165$, $p=0.004$ ). A statistically significant relationship was also found between prolonged operative time and increased VAS score $(r=0.140, p=0.015)$ (Table VI).

Mean operative times, according to the consecutive case series, are shown in Figure 1. The row group variable is the version of the operations divided into 10 groups. In other words, column 1 shows the average duration of the first 10 operations, and column 2 shows the average duration of operations 11-20. Op id A, B, and C refer to Surgeon A, B, and C, respectively. Based on this, the mean operative time started to stabilise under $40 \mathrm{~min}$, and SD decreased: 51 cases for Surgeon A, 71 cases for Surgeon B, and 81 cases for Surgeon $C$ were determined (SD from 11.00 to 6.8 , SD from 11.6 to 7.2, SD from 12 to 7.4, respectively). It can be said that the operative times of Surgeon A were the shortest. When the groups are evaluated together, the mean operative time for the first 60 cases was $79.7 \pm 23 \mathrm{~min}$, and after the $60^{\text {th }}$ case it was $36.7 \pm 9.7 \mathrm{~min}(p<0.001)$.

\section{Discussion}

This study showed that three surgeons with similar backgrounds should experience 51-81 cases for 
Table II. Comparison of the groups in terms of clinical characteristics and results

\begin{tabular}{|c|c|c|c|c|}
\hline Parameter & $\begin{array}{l}\text { A }(n=103) \\
\text { Mean } \pm \text { SD }\end{array}$ & $\begin{array}{c}\mathrm{B}(n=98) \\
\text { Mean } \pm \text { SD }\end{array}$ & $\begin{array}{l}C(n=98) \\
\text { Mean } \pm \text { SD }\end{array}$ & $P$-value \\
\hline Age & $40.61 \pm 14.44$ & $41.71 \pm 13.55$ & $41.48 \pm 13.17$ & $0.835^{a}$ \\
\hline BMI & $25.14 \pm 3.67$ & $25.53 \pm 4.02$ & $25.68 \pm 3.65$ & $0.573^{a}$ \\
\hline Operative time & $54.35 \pm 26.76$ & $64.73 \pm 27.18$ & $69.33 \pm 29.18$ & $0.001^{a, \star \star}$ \\
\hline VAS $^{\ddagger}$ & $3(2,4)$ & $4(3,4)$ & $4(3,5)$ & $<0.001^{\mathrm{b}, * \star}$ \\
\hline Length of stay ${ }^{\ddagger}$ [days] & $1(1,2)$ & $1(1,2)$ & $1(1,2)$ & $0.259^{b}$ \\
\hline Follow-up time [months] & $24.78 \pm 1.91$ & $18.55 \pm 2.00$ & $10.60 \pm 2.40$ & $<0.001^{a, * *}$ \\
\hline Parameter & $n(\%)$ & $n(\%)$ & $n(\%)$ & $P$-value \\
\hline Sex: & & & & $0.975^{c}$ \\
\hline Female & $27(26.2)$ & $26(26.5)$ & $27(27.6)$ & \\
\hline Male & $76(73.8)$ & $72(73.5)$ & $71(72.4)$ & \\
\hline Hernia type: & & & & $0.549^{d}$ \\
\hline Medial & $12(11.7)$ & $8(8.2)$ & $14(14.3)$ & \\
\hline Femoral & $1(1)$ & $1(1)$ & $4(4.1)$ & \\
\hline Lateral & $86(83.5)$ & $84(85.7)$ & 77 (78.6) & \\
\hline Combined & $4(3.9)$ & $5(5.1)$ & $3(3.1)$ & \\
\hline ASA: & & & & $0.158^{d}$ \\
\hline I & $85(82.5)$ & $85(86.7)$ & $83(84.7)$ & \\
\hline$\|$ & $18(17.5)$ & $12(12.2)$ & $11(11.2)$ & \\
\hline III & $0(0)$ & $1(1.0)$ & $4(4.1)$ & \\
\hline Conversion to open & $5(4.9)$ & $5(5.1)$ & $3(3.1)$ & $0.829^{d}$ \\
\hline Conversion to TAPP & $2(1.9)$ & $3(3.1)$ & $3(3.1)$ & $0.826^{d}$ \\
\hline Intraoperative complication & $8(7.7)$ & $6(6.1)$ & $5(5.1)$ & $0.913^{d}$ \\
\hline Postoperative haematoma & $3(2.9)$ & $4(4.1)$ & $4(4.1)$ & $0.868^{d}$ \\
\hline Postoperative seroma & $6(5.8)$ & $8(8.2)$ & $5(5.1)$ & $0.655^{c}$ \\
\hline Chronic pain & $2(1.9)$ & $2(2)$ & $2(2)$ & $0.999^{d}$ \\
\hline Early recurrence & $0(0)$ & $3(3.1)$ & $2(2)$ & $0.205^{d}$ \\
\hline Late recurrence & $5(4.9)$ & $4(4.1)$ & $4(4.1)$ & $0.999^{d}$ \\
\hline
\end{tabular}

a One-way analysis of variance, ${ }^{b}$ Kruskal-Wallis test, 'Pearson's $\chi^{2}$ test, ${ }^{d}$ Fisher-Freeman-Halton exact test. ${ }^{\ddagger}$ Results represented as median (interquartile range). ${ }^{* *} p<0.01$.

an acceptable duration of surgery in the TEP learning curve under the same conditions. We observed that the rate of conversion to open surgery increases as the BMI increases, and the rate of intraoperative complications and recurrence is higher in elderly patients. In addition, we found that there was a positive correlation between increased operative time and postoperative pain.
The studies about the TEP learning curve without the supervision of experienced surgeons are limited in the literature. Lau et al. [17] reported that after 80 cases, the mean operative time started to stabilise, and the conversion rate was $1.6 \%$, and they did not detect recurrence. Recurrent cases constituted 10\% of the population in the same study. In another similar study, Mathur et al. [18] showed that the mean 
Table III. Risk factors affecting conversion to open surgery and comparison of groups in terms of BMI

\begin{tabular}{|c|c|c|c|}
\hline \multirow[t]{2}{*}{ Parameter } & Conversion (-) & Conversion (+) & \multirow[t]{2}{*}{$P$-value } \\
\hline & Median (Q1, Q3) & Median (Q1, Q3) & \\
\hline Age $^{\ddagger}$ & $39.5(29,51)$ & $42(31,47)$ & 0.697 \\
\hline $\mathrm{BMI}^{\ddagger}:$ & $25.25(22.4,28.3)$ & $30.4(26,31.2)$ & $0.004^{\star *}$ \\
\hline Surgeon A & $24.4(22,28)$ & $31(25.7,31.2)$ & 0.110 \\
\hline Surgeon B & $25.3(22.4,28.4)$ & $29.8(26,30.4)$ & 0.252 \\
\hline Surgeon C & $25.6(22.5,28.4)$ & $30.4(29.3,32.4)$ & $0.009^{* *}$ \\
\hline Parameter & $n(\%)$ & $n(\%)$ & $P$-value \\
\hline Sex: & & & $0.525^{f}$ \\
\hline Female & $78(97.5)$ & $2(2.5)$ & \\
\hline Male & $208(95)$ & $11(5)$ & \\
\hline Hernia type: & & & $0.853^{d}$ \\
\hline Medial & $32(94.1)$ & $2(5.9)$ & \\
\hline Femoral & $6(100)$ & $0(0)$ & \\
\hline Lateral & $236(95.5)$ & $11(4.5)$ & \\
\hline Combined & $12(100)$ & $0(0)$ & \\
\hline
\end{tabular}

${ }^{e}$ Mann-Whitney $U$ test, ${ }^{c}$ Pearson's $\chi^{2}$ test, ${ }^{d}$ Fisher-Freeman-Halton exact test, ${ }^{f}$ Fisher's exact test. ${ }^{\ddagger}$ Results presented as median (interquartile range). ${ }^{* *} p<0.01$.

Table IV. Evaluation of factors associated with intraoperative complications and comparison of the groups in terms of age

\begin{tabular}{|c|c|c|c|}
\hline \multirow[t]{2}{*}{ Parameter } & Intraoperative complications (-) & Intraoperative complications (+) & \multirow[t]{2}{*}{$P$-value } \\
\hline & Median (Q1, Q3) & Median (Q1, Q3) & \\
\hline Age $^{\ddagger}:$ & $39(29,50)$ & $58(47,60)$ & $<0.001^{\star *}$ \\
\hline Surgeon A & $38(28,48)$ & $55(44.5,60.5)$ & $0.018^{\star}$ \\
\hline Surgeon B & $39.5(29,51)$ & $54.5(46,60)$ & $0.030^{*}$ \\
\hline Surgeon C & $39(29,48)$ & $58(54,67)$ & $0.003^{* \star}$ \\
\hline $\mathrm{BMI}^{\ddagger}$ & $25.6(22.5,28.4)$ & $27(23,29.4)$ & 0.068 \\
\hline Parameter & $n(\%)$ & $n(\%)$ & $P$-value \\
\hline Sex: & & & $0.562^{c}$ \\
\hline Female & $76(95)$ & $4(5)$ & \\
\hline Male & $204(93.2)$ & $15(6.8)$ & \\
\hline Hernia type: & & & $0.065^{d}$ \\
\hline Medial & $28(94.1)$ & $6(17.6)$ & \\
\hline Femoral & $6(100)$ & $0(0)$ & \\
\hline Lateral & $234(97.2)$ & $13(5.3)$ & \\
\hline Combined & $12(100)$ & $0(0)$ & \\
\hline
\end{tabular}

${ }^{e}$ Mann-Whitney U test, ${ }^{c P e a r s o n}$ 's $\chi^{2}$ test, ${ }^{d}$ Fisher-Freeman-Halton exact test. ${ }^{\ddagger}$ Results presented as median (interquartile range). ${ }^{*} p<0.05,{ }^{* *} p<0.01$. 
Table V. Evaluation of factors affecting late recurrence and comparison of the groups in terms of age

\begin{tabular}{|c|c|c|c|}
\hline \multirow[t]{2}{*}{ Parameter } & Late recurrence $(-)$ & Late recurrence $(+)$ & \multirow[t]{2}{*}{$P$-value } \\
\hline & Median (Q1, Q3) & Median (Q1, Q3) & \\
\hline Age $^{\ddagger}:$ & $39(29,51)$ & $58(42,62)$ & $0.008^{\star *}$ \\
\hline Surgeon A & $39(29,48)$ & $62(42,67)$ & $0.028^{*}$ \\
\hline Surgeon B & $41(29,51)$ & $43(40,52)$ & 0.504 \\
\hline Surgeon C & $39(30,51)$ & $60(43.5,62)$ & 0.102 \\
\hline $\mathrm{BMI}^{\ddagger}$ & $25.5(22.4,28.4)$ & $23.4(21.3,28.2)$ & 0.445 \\
\hline Parameter & $n(\%)$ & $n(\%)$ & $P$-value \\
\hline Sex: & & & $0.196^{f}$ \\
\hline Female & $79(98.8)$ & $1(1.3)$ & \\
\hline Male & $207(94.5)$ & $12(5.5)$ & \\
\hline Hernia type: & & & $0.488^{d}$ \\
\hline Medial & $31(91.2)$ & $3(8.8)$ & \\
\hline Femoral & $6(100)$ & $0(0)$ & \\
\hline Lateral & $237(96)$ & $10(4)$ & \\
\hline Combined & $12(100)$ & $0(0)$ & \\
\hline
\end{tabular}

${ }^{e}$ Mann-Whitney $U$ test, ${ }^{c}$ Pearson's $\chi^{2}$ test, ${ }^{d}$ Fisher-Freeman-Halton exact test, ${ }^{f}$ Fisher's exact test. ${ }^{\ddagger}$ Results presented as median (interquartile range). ${ }^{* *} p<0.01$.

Table VI. Evaluation of factors associated with the operative time

\begin{tabular}{|lcc|}
\hline Parameter & \multicolumn{2}{c|}{ Operative time } \\
\cline { 2 - 3 } & $r$ & $P$-value \\
\hline Age & -0.029 & 0.619 \\
\hline BMI & 0.165 & $0.004^{* *}$ \\
\hline VAS & 0.140 & $0.015^{*}$ \\
\hline Parameter & Mean \pm SD & $P$-value \\
\hline Sex: & & $0.510^{8}$ \\
\hline Female & $64.45 \pm 27.07$ & \\
\hline Male & $62.01 \pm 28.81$ & \\
\hline- Pearson's correlation coefficients, ${ }^{\text {ind }}$ & \\
\hline
\end{tabular}

operative time stabilised after 18 cases, but there was an increase again between 51 and 61 cases, and they also indicated the conversion rate as $1.3 \%$ and the recurrence rate as $2 \%$. In the same study, only irreducible hernias were excluded, and patients who had bilateral, recurrent, and abdominal surgery were included. In a study conducted on a single inexpe- rienced surgeon without another supervision, Choi et al. [14] reported that the operative time stabilised after 60 cases, with a conversion rate of $0.8 \%$ and recurrence rate of $0.4 \%$. In the same study, only bilateral cases were excluded - patients who had abdominal surgery and recurrence were included in the study. On the other hand, Schouten et al. [19] predicted that the procedure could be performed safely after 50 cases in a study comparing surgeons with different experiences. However, they stated that the most significant decrease in the conversion rate was after 250 cases. We think that the difference in patient selection criteria creates the difficulty in evaluating these studies objectively in terms of the learning curve. Only patients fulfilling predetermined criteria were included in our study. In the evaluation of the learning curve, the exclusion of factors (bilateral hernias, recurrent hernias, irreducible hernias, giant scrotal hernias, and patients with a history of abdominal surgery) that could affect the results partially led to the formation of more homogeneous groups. Our conversion rate decreased statistically after the first 60 cases. Similarly, our recurrence rate of $6 \%$ decreased to $1.4 \%$ after the first 60 cases, but 
A

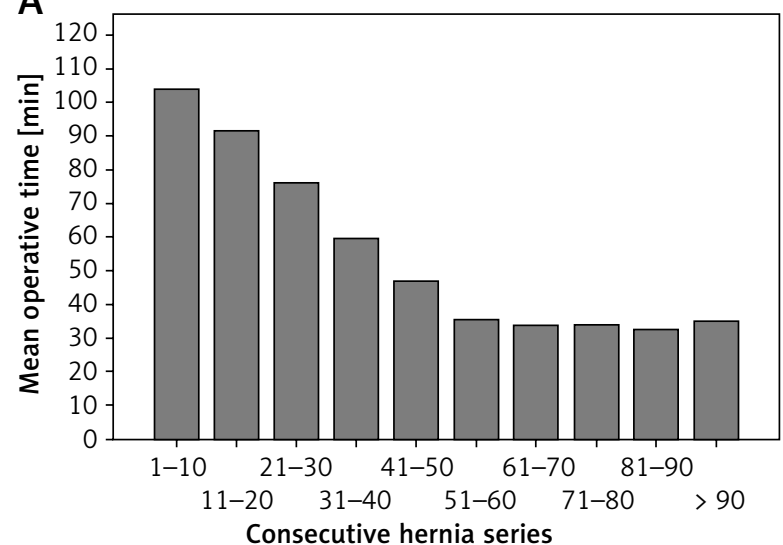

C

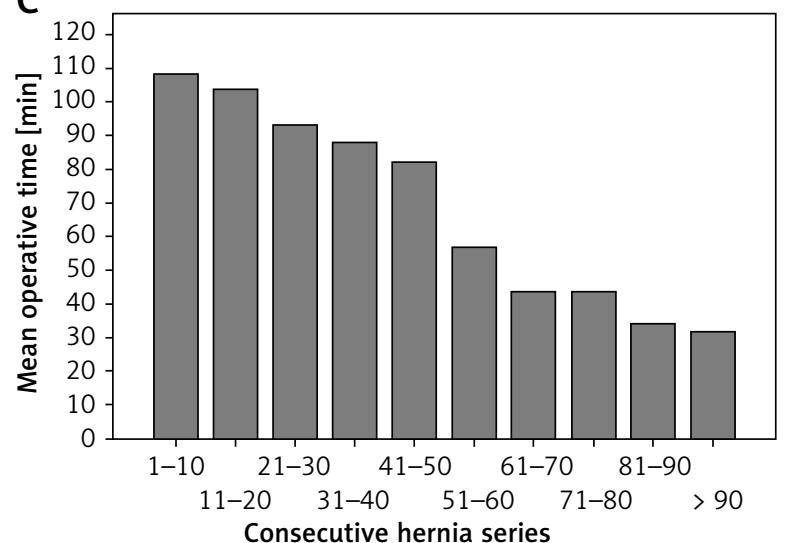

B

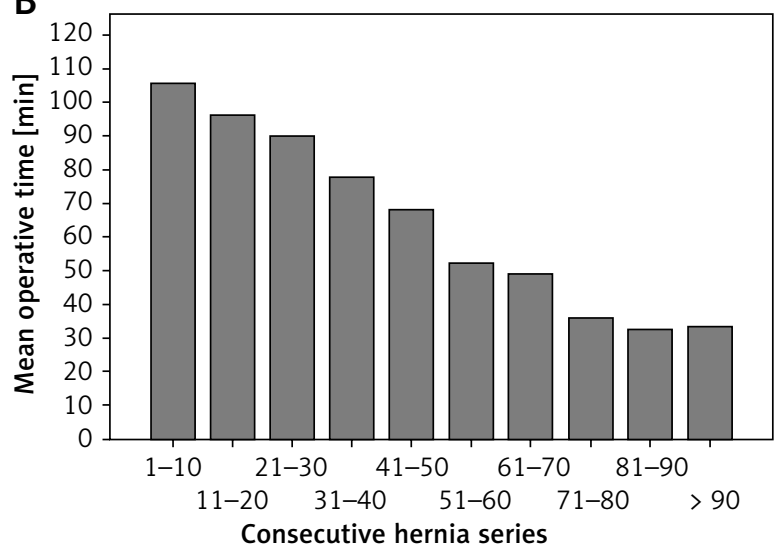

D

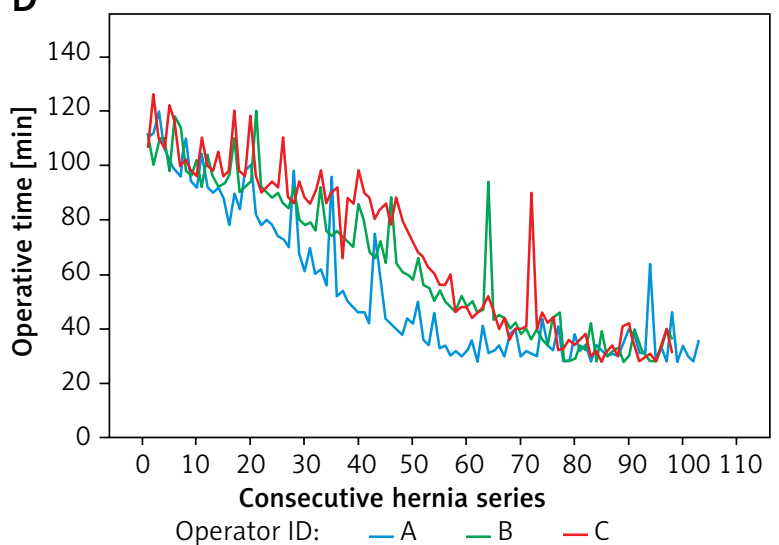

Figure 1. Comparison of groups (Surgeon A (A), B (B), C (C)) in terms of mean operative time according to consecutive case series (D)

this was not reflected in the statistics due to the low number of recurrent cases. There was no difference between the groups in terms of both recurrence and conversion. In the similar studies mentioned above $[14,17,18]$, it was stated that neither recurrence nor conversion rates decreased with experience. In contrast, in the retrospective analysis of the 1682 TEP procedure, Lamb et al. [20] stated that in the first 80 cases the recurrence rate was $2 \%$, and it was $1 \%$ for surgeons with experience of more than 200 TEPs. When compared with these studies, we see that both our conversion and recurrence rates are comparable only after experience.

Female gender, abdominal surgery history, and scrotal hernia are among the risk factors for conversion [14, 21]. In our study, only BMI increase was observed to increase the conversion rate. Specifically, we found that obesity (BMI $\left.\geq 30 \mathrm{~kg} / \mathrm{m}^{2}\right)$ increased the conversion rate nine-fold. At the same time, it is noteworthy that all patients converted due to the in- ability to enter the preperitoneal space were obese. There are several possible causes for this. First of all, in obese patients, the excess of subcutaneous fat tissue may cause more bleeding during the entry into the preperitoneal space, resulting in failure to enter the correct area or entering the wrong area. Second, the excess of intra-abdominal fatty tissue and omentum may further limit the already narrow working area. Therefore, selecting thinner patients during the learning curve period may provide a safer and less disappointing experience in this period.

Patient-related factors for recurrence were examined in the systematic review and meta-analysis by Burcharth et al. [22], in which it was stated that female gender, $\mathrm{BMI}>30 \mathrm{~kg} / \mathrm{m}^{2}$, direct inguinal hernia, and smoking are risk factors. Age was the only risk factor for recurrence in our study. Elderly patients (age $\geq 60$ years) had six-fold more recurrences. One of the reasons for this may be impaired wound healing in elderly patients [23]. The higher rates of peri- 
toneal tear in elderly patients may be another reason for the higher rate of recurrence in this age group. It has been stated that peritoneal tear may be associated with recurrence [24]. However, this relationship is not clear and needs to be studied further. Unlike in the literature, no other patient-related risk factors were detected for both recurrence and conversion.

In addition to organ injuries and vascular injuries, minor complications such as peritoneal tear can be seen during TEP. Zhu et al. [25] reported an intraoperative complication rate of $3 \%$ in patients undergoing TEP in their meta-analysis. In another study conducted with 3482 TEP patients, the rate of intraoperative complications was $1.6 \%$, and a history of abdominal surgery and scrotal or bilateral inguinal hernia were found to be independent risk factors for intraoperative complications [21]. In our study, intraoperative bladder injury occurred in 1 patient during dissection. After primary repair with laparoscopic 3/0 Vicryl, the operation was completed with the TEP method, and no complication was observed in the patient, who was followed up with a foley catheter 5 days postoperatively. Other intraoperative complications were all peritoneal tears requiring sutures. With increased experience (after the first 60 cases), intraoperative complications were similarly reduced for all three surgeons.

Old age was determined as a risk factor for intraoperative complications, and it was observed that the risk was five-fold higher in elderly patients. Thinner parietal peritoneum in elderly patients [26] may increase the likelihood of peritoneal tear during dissection. Peritoneal tear leads to pneumoperitoneum that narrows the pre-peritoneal working area, which increases the risk of conversion. In our study, more than half $(55 \%)$ of the patients with peritoneal tear required conversion. Therefore, more careful dissection is recommended, especially in elderly patients.

In previous studies on the learning curve [14, 18], postoperative complications have been reported in the range $7.3 \%$ to $26 \%$. Krishna et al. [27] reported a $22 \%$ postoperative complication rate in surgeons with at least 15 years of TEP experience. We would like to state that the $10 \%$ postoperative complication rate in our study did not decrease with experience for any of the three surgeons.

Studies show that younger age and female gender are associated with pain after laparoscopic inguinal hernia repair [28]. Unlike in the literature, no relationship was found between pain and age and gender in our study. In a study by Mitura et al. [29], it was shown that glue fixation significantly reduces postoperative pain. On the other hand, in another randomised controlled study, fixation techniques (self-gripping mesh, lightweight mesh, and lightweight mesh with fixation) were compared, and no difference was found regarding postoperative pain [30]. A similar mesh was used in our study, and a laparoscopic tucker was routinely used for patch fixation. Therefore, it was possible to partially control the mesh-related factors in postoperative pain assessment. Interestingly, there was a positive correlation between postoperative pain and prolonged operative time. Hence, the VAS score in the Surgeon A group, which had a shorter operative time, was observed to be lower than the Surgeon B and C groups. Although there is not enough evidence to explain this situation, the prolongation of the operative time may have led to more tissue manipulation that caused more damage to the nerve fibres. Based on this, we can infer that the increase in experience, and thus the decrease in the operative time, also affects postoperative pain. However, we think that the current situation should be examined further. There was no difference in the rate of chronic groin pain related to experience.

This study has some limitations. First of all, this was a retrospective study. Although different follow-up times between surgeons do not affect our complications and the rate of conversion to open surgery, it is especially important in affecting the recurrence rate. Secondly, sexual functioning and quality of life were not questioned. On the other hand, it can be considered a strength of the study that most patients regularly attended control visits, and the records were collected prospectively.

All three surgeons shared a common opinion that one of the most critical factors that prolongs the operative time during the learning curve is the effort required to enter the correct plane under the umbilicus. Based on their experience, they stated that it was more challenging to get into the correct plane in patients with high BMI, and peritoneal tear was more frequent during entry in elderly patients. We would like to emphasise that $90 \%$ of our study's conversions were due to the inability to enter the preperitoneal space and peritoneal tear. Based on the results of this study, we think that the selection of thinner and younger patients will decrease the complication rates, especially during the learning curve period. 
Surgeons have some concerns about laparoscopic inguinal hernia repair; steep learning curve and lack of knowledge are among them. In addition, in a survey study conducted on surgeons in Poland, it was shown that financial concerns also affect the orientation to laparoscopic surgery. It is also widely believed that it should only be performed by surgeons with advanced skills in minimally invasive surgery [31]. In another study, it was reported that the laparoscopic skills of surgeons who had access to laparoscopic training were more satisfactory [32]. In particular, making laparoscopic courses a part of education is important for reducing surgeons' concerns. In our study, surgeons were certified by attending courses (theoretical training and practical training with a surgical simulator) organised by national surgical associations. In addition, they only participated in the operations of surgeons experienced in TEP hernia repair as assistants. This study is encouraging in that it shows that surgeons willing to perform TEP hernia repair can complete their learning curve on their own provided they receive adequate training.

\section{Conclusions}

In this study, a plateau was reached during the operation period after 51-81 cases. Acceptable results were obtained after 60 cases for conversion, intra-operative complications, and recurrence. Based on the data of this study, selecting relatively thin $\left(\mathrm{BMI}<30 \mathrm{~kg} / \mathrm{m}^{2}\right)$ and younger (age $<60$ years) patients at the beginning of the learning curve can be recommended both for patient safety and to allow surgeons to experience this period more comfortably.

\section{Conflict of interest}

The authors declare no conflict of interest.

\section{References}

1. Ger R. The management of certain abdominal herniae by intraabdominal closure of the neck of the sac. Ann R Coll Surg Engl 1982; 64: 342-4.

2. Ger R, Monroe K, Duvivier R, Mishrick A. Management of indirect inguinal hernias by laparoscopic closure of the neck of the sac. Am J Surg 1990; 159: 370-3.

3. Ferzli GS, Massad A, Albert P. Extraperitoneal endoscopic inguinal hernia repair. J Laparoendosc Surg 1992; 2: 281-6.

4. Dulucq JL, Wintringer P, Mahajna A. Laparoscopic totally extraperitoneal inguinal hernia repair: lessons learned from 3,100 hernia repairs over 15 years. Surg Endosc 2009; 23: 482-6.
5. Wakasugi M, Nakahara Y, Hirota M, et al. Learning curve for single incision laparoscopic totally extraperitoneal inguinal hernia repair. Asian J Endosc Surg 2019; 12: 301-5.

6. Kassir R, Tiffet O, Bourbon M, et al. Laparoscopic hernia repair with 3-millimeter instruments: a point of technique and illustrative case video. Surg Innov 2015; 22: 366-7.

7. Putnis S, Berney CR. Totally extraperitoneal repair of inguinal hernia: techniques and pitfalls of a challenging procedure. Langenbecks Arch Surg 2012; 397: 1343-51.

8. Meyer A, Blanc P, Balique JG, et al. Herniorrafia inguinal laparoscópica totalmente extraperitoneal: vinte e sete complicações graves após 4565 operações consecutivas. Rev Col Bras Cir 2013; 40: 32-6.

9. Meyer A, Blanc P, Kassir R, Atger J. Laparoscopic hernia: umbilical-pubis length versus technical difficulty. JSLS 2014; 18: e2014.00078.

10. Lal P, Kajla RK, Chander J, Ramteke VK. Laparoscopic total extraperitoneal (TEP) inguinal hernia repair: overcoming the learning curve. Surg Endosc 2004; 18: 642-5.

11. Ebbinghaus $\mathrm{H}$ (1885). Memory: a contribution to experimental psychology. Ann Neurosci 2013; 20: 155-6.

12. Artibani W, Novara G. Cancer-related outcome and learning curve in retropubic radical prostatectomy: "If You need an operation, the most important step is to choose the right surgeon." Eur Urol 2008; 53: 874-6.

13. Lim JW, Lee JY, Lee SE, et al. The learning curve for laparoscopic totally extraperitoneal herniorrhaphy by moving average. J Korean Surg Soc 2012; 83: 92-6.

14. Choi YY, Kim Z, Hur KY. Learning curve for laparoscopic totally extraperitoneal repair of inguinal hernia. Can J Surg 2012; 55: 33-6.

15. Suguita FY, Essu FF, Oliveira LT, et al. Learning curve takes 65 repetitions of totally extraperitoneal laparoscopy on inguinal hernias for reduction of operating time and complications. Surg Endosc 2017; 31: 3939-45.

16. Miserez M, Alexandre JH, Campanelli G, et al. The European hernia society groin hernia classification: simple and easy to remember. Hernia 2007; 11: 113-6.

17. Lau H, Patil NG, Yuen WK, Lee F. Learning curve for unilateral endoscopic totally extraperitoneal (TEP) inguinal hernioplasty. Surg Endosc 2002; 16: 1724-8.

18. Mathur S, Lin SYS. The learning curve for laparoscopic inguinal hernia repair: a newly qualified surgeon perspective. J Surg Res 2016; 205: 246-51.

19. Schouten N, Simmermacher RKJ, van Dalen T, et al. Is there an end of the "learning curve" of endoscopic totally extraperitoneal (TEP) hernia repair? Surg Endosc 2013; 27: 789-94.

20. Lamb ADG, Robson AJ, Nixon SJ. Recurrence after totally extraperitoneal laparoscopic repair: Implications for operative technique and surgical training. Surgeon 2006; 4: 299-307.

21. Schouten N, Elshof JWM, Simmermacher RKJ, et al. Selecting patients during the "learning curve" of endoscopic totally extraperitoneal (TEP) hernia repair. Hernia 2013; 17: 737-43.

22. Burcharth J, Pommergaard HC, Bisgaard T, Rosenberg J. Patient-related risk factors for recurrence after inguinal hernia repair: a systematic review and meta-analysis of observational studies. Surg Innov 2015; 22: 303-17. 
23. Thomas Hess C. Checklist for factors affecting wound healing. Adv Skin Wound Care 2011; 24: 192.

24. Lau H. Endoscopic totally extraperitoneal inguinal hernioplasty for recurrence after open repair. ANZ J Surg 2004; 74: 877-80.

25. Zhu X, Cao H, Ma Y, et al. Totally extraperitoneal laparoscopic hernioplasty versus open extraperitoneal approach for inguinal hernia repair: a meta-analysis of outcomes of our current knowledge. Surgeon 2014; 12: 94-105.

26. Schaefer B, Bartosova M, Macher-Goeppinger S, et al. Quantitative histomorphometry of the healthy peritoneum. Sci Rep 2016; 6: 21344

27. Krishna A, Bansal VK, Misra MC, et al. Totally extraperitonea repair in inguinal hernia: more than a decade's experience at a tertiary care hospital. Surg Laparosc Endosc Percutan Techn 2019; 29: 247-51.

28. Tolver MA, Rosenberg J, Bisgaard T. Early pain after laparoscopic inguinal hernia repair. A qualitative systematic review. Acta Anaesthesiol Scand 2012; 56: 549-57.

29. Mitura K, Garnysz K, Wyrzykowska D, Michatek I. The change in groin pain perception after transabdominal preperitoneal inguinal hernia repair with glue fixation: a prospective trial of a single surgeon's experience. Surg Endosc 2018; 32: 4284-9.

30. Pielaciński K, Puła B, Wróblewski T, et al. Totally extraperitoneal inguinal hernia repair with or without fixation leads to similar results. Outcome of randomized prospective trial. Videosurgery Miniinv 2020; 15: 1-10.

31. Mitura K, Dąbrowiecki S, Śmietański M, et al. The experience and awareness of laparoendoscopic procedures among Polish surgeons in everyday clinical practice. Videosurgery Miniinv 2017; 12: 13-8.

32. Brzoszczyk B, Milecki T, Jarzemski P, et al. Urology resident training in laparoscopic surgery: results of the first national survey in Poland. Videosurgery Miniinv 2019; 14: 433-41.

Received: 19.08.2020, accepted: 11.10. 2020. 